\title{
Real-time Forecasting of the 2014 Dengue Fever Season in Thailand
}

\author{
Nicholas G. Reich ${ }^{1,2}$, Krzysztof Sakrejda1, Stephen A. Lauer*1, Derek A. Cummings ${ }^{2}$, \\ Paphanij Suangtho ${ }^{3}$, Soawapak Hinjoy ${ }^{2,3}$, Sopon lamsirithaworn ${ }^{3}$, Hannah Clapham², \\ Henrik Salje ${ }^{2}$ and Justin Lessler ${ }^{2}$
}

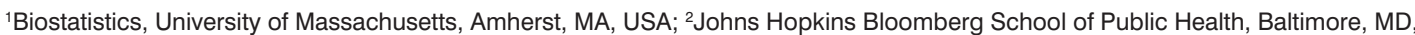
USA; ${ }^{3}$ Thai Ministry of Public Health and Bureau of Epidemiology, Nonthaburi, Thailand

\section{Objective}

To develop a statistical model for dengue fever surveillance that uses data from across Thailand to give early warning of developing epidemics.

\section{Introduction}

Dengue is a major cause of morbidity in Thailand. Annual outbreaks of varying sizes provide a particular challenge to the public health system because treatment of severe cases requires significant resources. Advanced warning of increases in incidence could help public health authorities allocate resources more effectively and mitigate the impact of epidemics.

\section{Methods}

Starting in November of 2013, we received biweekly dengue fever count data from the Thai Ministry of Public Health and Bureau of Epidemiology. After cleaning, this data was combined with historical case counts dating back to 1968 and seasonal trends for each province to build a series of statistical models, ranging from smoothed regression-based models to Gaussian process models. Once case counts are completed for a given biweek - which can take up to six months in rural provinces - each model can be cross-validated and compared, using methods such as mean average squared error (MASE).

\section{Results}

Thus far we have generated forecasts at 22 time points for a suite of different models. Since cases continue to be reported throughout 2014 , the evaluation of the prediction performance is ongoing.

\section{Conclusions}

One of the largest challenges from real-time reporting is dealing with incomplete case count data. We have found that we do not receive a full case count for a province in a given biweek for at least two months and often up to six months. Since this is the first year of collected data, we have found it difficult to decipher whether case count differences are attributable to reporting rates, seasonality, climate change, or other population factors. Through working on this project, we have learned that models built on historical data are not necessarily applicable in real-time and that novel methods must be employed to improve infectious disease predictions, especially in developing countries with partial reporting data.

\section{Keywords}

Infectious disease surveillance; Real-time forecasts; Statistical model selection; Dengue fever

\section{*Stephen A. Lauer}

E-mail: stephenalauer@gmail.com 